\title{
Low-Cost Sensing with Ring Oscillator Arrays for Healthier Reconfigurable Systems
}

\author{
KENNETH M. ZICK, University of Southern California Information Sciences Institute \\ JOHN P. HAYES, University of Michigan
}

\begin{abstract}
Electronic systems on a chip increasingly suffer from component variation, voltage noise, thermal hotspots, and other subtle physical phenomena. Systems with reconfigurability have unique opportunities for adapting to such effects. Required, however, are low-cost, fine-grained methods for sensing physical parameters. This article presents powerful, novel approaches to online sensing, including methods for designing compact reconfigurable sensors, low-cost threshold detection, and several enhanced measurement procedures. Together, the approaches help enable systems to autonomously uncover a wealth of physical information. A highly efficient counter and improved ring oscillator are introduced, enabling an entire sensor node in just 8 Virtex-5 LUTs. We describe how variations can be measured in delay, temperature, switching-induced IR drop, and leakage-induced IR drop. We demonstrate the proposed approach with an experimental system based on a Virtex-5, instrumented with over 100 sensors at an overhead of only $1.3 \%$. Results from thermally controlled experiments provide some surprising insights and illustrate the utility of the approach. Online sensing can help open the door to physically adaptive computing, including fine-grained power, reliability, and health management schemes for systems on a chip.
\end{abstract}

Categories and Subject Descriptors: C.4 [Performance of Systems]: Fault tolerance, Measurement techniques; B.8.1 [Performance and Reliability]: Reliability, Testing, and Fault Tolerance

General Terms: Design, Measurement, Performance, Reliability

Additional Key Words and Phrases: Sensing, FPGAs, process variation, voltage, power, leakage, temperature, wearout, ring oscillator, health management, physically-adaptive computing

ACM Reference Format:

Zick, K. M. and Hayes, J. P. 2012. Low-cost sensing with ring oscillator arrays for healthier reconfigurable systems. ACM Trans. Reconfig. Technol. Syst. 5, 1, Article 1 (March 2012), 26 pages.

DOI $=10.1145 / 2133352.2133353$ http://doi.acm.org/10.1145/2133352.2133353

\section{INTRODUCTION}

As semiconductor technology advances, electronic systems are suffering from increasing physical difficulties. The amount of manufacturing variation in transistors and interconnect is growing dramatically [ITRS 2011], resulting in chips with many marginal regions and components. The threat worsens even further at the lower supply voltages needed for low-power operation [Dighe et al. 2010]. Combined with lifetime wearout and variations in application activity, this is leading to excessive power consumption, thermal hotspots, and reduced performance and reliability. Given their fine-grained reconfigurability, FPGA-based systems have a unique potential for both measuring and

This work was supported in part by a NASA Langley Research Center GSRP Fellowship and by the National Science Foundation under grant CCF-1017142.

Authors' addresses: K. M. Zick, University of Southern California Information Sciences Institute, Arlington, VA; email: kzick@isi.edu; J. P. Hayes, Department of Computer Science \& Engineering, University of Michigan, Ann Arbor, MI.

Permission to make digital or hard copies of part or all of this work for personal or classroom use is granted without fee provided that copies are not made or distributed for profit or commercial advantage and that copies show this notice on the first page or initial screen of a display along with the full citation. Copyrights for components of this work owned by others than ACM must be honored. Abstracting with credit is permitted. To copy otherwise, to republish, to post on servers, to redistribute to lists, or to use any component of this work in other works requires prior specific permission and/or a fee. Permissions may be requested from Publications Dept., ACM, Inc., 2 Penn Plaza, Suite 701, New York, NY 10121-0701 USA, fax +1 (212) 869-0481, or permissions@acm.org.

(c) 2012 ACM 1936-7406/2012/03-ART1 $\$ 10.00$

DOI 10.1145/2133352.2133353 http://doi.acm.org/10.1145/2133352.2133353 
managing physical effects. While electronic systems must typically compute precise results, reconfigurable systems can in principle change how they compute. We refer to such a paradigm as physically adaptive computing [Zick and Hayes 2010c].

A traditional approach to handling physical variation is to estimate worst-case conditions and to add extra margin into a system. However, this is becoming increasingly suboptimal as the amount of variation soars. Moreover, it is becoming harder to accurately model and simulate the resulting subtle and complex physical interactions. One alternative approach is to map the variations of every chip before deployment and for system implementations to be customized to each chip. Proposals for such "chipwise" customization are presented in Srinivasan and Narayanan [2006] and Cheng et al. [2006], using variation-aware place and route. However, manufacturers do not yet have the incentive to generate and provide detailed variation maps, and only in specialized cases can a system-maker or user shoulder the burden of chipwise testing, $\mathrm{CAD}$, and bitstream handling. Moreover, a pre-deployment approach cannot help with infant mortality, changes in workload, or other parameter shifts that occur in the field.

What is required, therefore, is a low-cost, online method for making fine-grained physical measurements in FPGA systems. Relying on fixed, built-in analog sensors is not an option for FPGA-based systems. While FPGA platforms often include a single analog temperature and voltage sensor, they do not provide a means of measuring a variety of quantities at arbitrary locations. Thus the challenge is to use the standard digital logic in a reconfigurable fabric to measure as many key physical parameters as possible, and as accurately and unobtrusively as possible.

The key research questions that need to be addressed include the following. What specific sensing capabilities are feasible with low overhead, and how low is "low"? Which types of physical parameters can be estimated? How are new online sensing methods useful for FPGA-based systems, and other types of systems? This article addresses all of these questions, and makes the following contributions:

- the design of a highly flexible sensor node implementable in reconfigurable logic and roughly four times smaller than the previous smallest design;

-improved procedures for inferring fine-grained variations in delay, temperature, switching-induced IR drop, and a previously hidden parameter: leakage-induced IR drop;

- several new low-overhead sensing capabilities such as a method for real-time comparison of ring oscillator frequencies to two threshold values;

- a case study of a Virtex-5-based experimental system instrumented with over 100 sensors, including results and insights from thermally-controlled experiments.

This article significantly extends our work from Zick and Hayes [2010a]. The remainder of the article is structured as follows. We provide background material and survey related work in Section 2. We then introduce new approaches to designing lowcost sensor nodes and discuss sensing capabilities in Section 3. Methods for estimating specific physical parameters are given in Section 4 . We present experimental results in Section 5. The implications of this research are discussed in Section 6, followed by conclusions in Section 7.

\section{BACKGROUND AND RELATED WORK}

\subsection{Background/Motivation}

Physical phenomena are having a growing impact as semiconductor technology continues to scale. Phenomena of interest include inherent variations in components (transistors, interconnect) as well as nonuniformities in operating conditions such as temperature and supply voltage. Component variations result from the manufacturing 


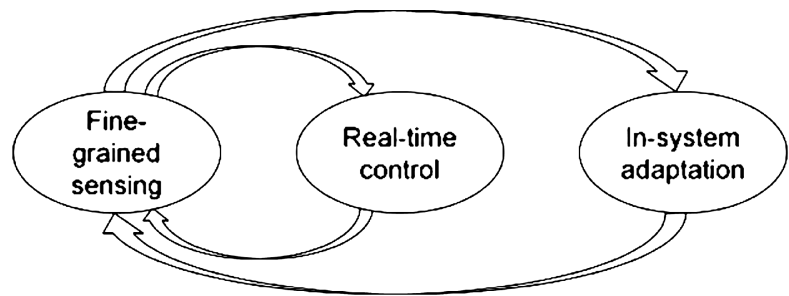

Fig. 1. Fine-grained sensing can be used concurrently for real-time control and for in-system adaptation.

process and also lifetime wearout. Variations within a die (intra-die) are in part spatially-correlated and in part stochastic from component to component.

A brief consideration of some relevant application domains helps to motivate finegrained on-line sensing. Many embedded systems are required to exhibit low power, high reliability, and high autonomy. Examples include the FPGA-based systems deployed in spacecraft, UAVs, and similar systems, such as NASA's SpaceCube [Flatley 2010]. Certain physical effects can be handled with coarse-grained control techniques (e.g. chip-wide voltage/frequency scaling, body bias, or power gating), but extreme variation calls for fine-grained system adaptation as well (Figure 1). Online sensing followed by variation-aware reconfiguration can potentially improve embedded system efficiency and extend lifetimes.

Another relevant application domain is high-performance reconfigurable computing. As with embedded systems, there is a need for fine-grained sensing of power, temperature, and wearout. Note that high-performance FPGA coprocessors are increasingly created via high-level synthesis, meaning low-level physical effects such as hotspots may go untreated. In fact, there are proposals for performing lightweight circuit synthesis at runtime [Vahid et al. 2008]. The trend toward high-level design increases the need for handling low-level effects at runtime.

Reconfigurable platforms sometimes include an analog voltage sensor and temperature sensor connected to an A/D converter, such as with the Xilinx System Monitor [Xilinx 2009]. At least one FPGA type, the space-grade Virtex-5QV, lacks even a single built-in voltage/temperature sensor [Xilinx 2010], and yet it is targeted for applications with high autonomy and reliability, so user-level methods of sensing would be highly desirable. Most importantly, even for platforms with a built-in sensor, the sensor coverage is limited to a single, fixed location.

Ring oscillators have been widely used for sensing variations in delay. They have also been proposed for sensing temperature and voltage. The parameters of delay, temperature, and voltage are all related in traditional CMOS circuits. These variables have often been modeled with simple linear relationships such as delay $=\mathrm{a} V+\mathrm{b} T+\mathrm{c}$. If two of the three related quantities can be measured accurately, then the third can be inferred. This is typically how researchers have proposed using ring oscillators in reconfigurable fabrics. For instance, the ring oscillator delay is measured at a known voltage, and then a model is employed to estimate the temperature. The average frequency of a ring oscillator at location $(x, y)$ is generally a function of the local voltage and temperature at that location:

$$
\text { frequency }(x, y)=f(V(x, y), T(x, y)) \text {. }
$$

The traditional approach to ring oscillator-based sensing, for thermal sensing in particular, suffers from several limitations. One is that local supply voltages are often lower than intended due to currents in a power grid with nonzero resistance, a phenomenon called IR drop [Ajami et al. 2003]. We will distinguish between switching-induced IR drop associated with switching activity in the system, and leakage-induced IR drop 
associated with leakage current. We will define the local voltage at location $(x, y)$ as:

$$
V(x, y)=V d d-\left(\Delta V_{s w}+\Delta V_{\text {leak }}\right) .
$$

Supply voltages can also undergo rapid, high-frequency swings after dramatic changes in switching activity. We refer to these as voltage transients. They are also known as $L \frac{d i}{d t}$ events or voltage "droops" [Muhtaroglu et al. 2004]. IR drop and voltage transients can lead designers to add extra margin to the supply voltage, thereby increasing power consumption and accelerating wearout. They can also lead to timing errors.

The nature of IR drop and voltage transients in reconfigurable systems is a rich, largely unexplored area. In this domain, unlike with ASIC- or MPU-based systems, no one currently has the full picture. The semiconductor vendors do not have access to the application logic or runtime conditions. The users do not have access to basic details and models of the FPGA layout, transistors, and power distribution system. There is a need and opportunity for new and holistic methods of introspection, whereby reconfigurable systems gain physical information about themselves at fine spatial and temporal scales.

\subsection{Related Work}

An early paper by Quénot et al. [1991] proposes the use of distributed ring oscillators to measure the voltage and temperature profile across an entire chip. Works such as Krishnamoorthy and Detofsky [2007] describe associated measurement procedures. Regarding FPGAs in particular, there have been several attempts at on-line thermal sensing using ring oscillators [López-Buedo and Boemo 1997; Conn 2000; Chen et al. 2007; Jones et al. 2007]; unfortunately, these methods entail relatively high overhead, and are becoming less accurate since the temperature dependence of advanced reconfigurable logic (e.g., $65 \mathrm{~nm}$ ) increasingly is swamped by voltage noise. In this paper, we build upon the approaches mentioned above.

There has been little work on online measurement of FPGA parameters other than temperature. An example of off-line characterization of FPGA component delays is given in Sedcole and Cheung [2006]. While the present article is focused on correlated variation, an important and related issue is sensing stochastic variation; in Zick and Hayes [2010b] we address the sensing of stochastic variations in component single event upset susceptibility. There has been much work in the area of online testing for hard defects which are in a sense highly localized variations. Finally, the general problem of how a system can effectively sense its own physical parameters is being studied in other contexts such as autonomic data centers [Jiang and Parashar 2009] and self-modeling robotic systems [Bongard et al. 2006].

\section{PROPOSED SENSING HARDWARE AND CAPABILITIES}

To enable effective online adaptation for physical effects, an FPGA-based system needs to be instrumented with the ability to measure physical parameters. The nature of spatial and temporal variations in a reconfigurable system necessitates that measurements be relatively fine-grained, while system constraints dictate that the solution be low-cost and efficient. A key question is how instrumentation can be designed to achieve these conflicting goals.

One common type of on-chip digital sensor is based on a ring oscillator circuit that feeds a frequency counter. The ring oscillator acts as a test circuit sensitive to parameters such as temperature. The oscillator is enabled for some reference period and the number of pulses is counted. The measured frequency is then used to estimate the physical quantity of interest. The utility of such sensors for FPGA-based systems has been limited by their high sensitivity to supply voltage fluctuations, and by the associated hardware overhead. 


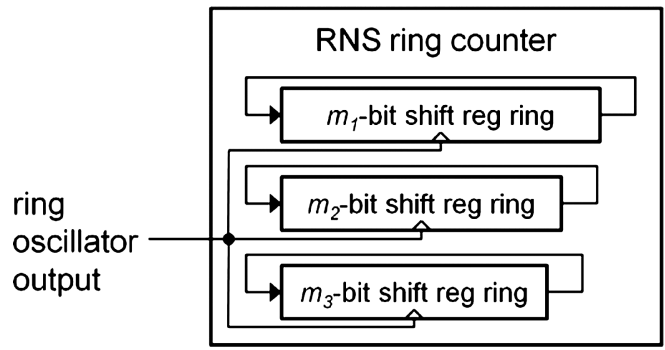

Fig. 2. Compact counter design using the residue number system (RNS). Up to two rings can be implemented with a single shift register LUT (SRL).

We now consider a much more efficient method of implementing the requisite frequency counters, as well as a compact implementation of a ring oscillator. We then describe a proposed sensor node and its methods of operation and access.

\subsection{Efficient Counting}

One approach to frequency counting is to implement a single centralized counter shared by multiple sensors [López-Buedo and Boemo 1997]. Aside from routing congestion, this approach allows only one sensor to be enabled at a time, which prevents a snapshot of a spatial profile from being captured. (A local parameter like voltage can change dramatically in the several milliseconds needed for serialized measurements.) Furthermore, certain parameters require a system to be paused during measurement, so serialization would cause a linear increase in the performance penalty. For these reasons, we need a compact counter that can be instantiated in each sensor and operated in parallel.

The goals for the counter design include not just compactness but also ease of use by the software that reads and decodes the sensor data. Traditionally these two goals have been mutually opposing, forcing a trade-off. A standard binary counter uses a binary representation for the count as the name implies; this format can be readily processed in software without special decoding. Unfortunately, a binary counter consumes a relatively large amount of hardware resources; a counter with period $M$ requires $\left\lceil\log _{2} M\right.$ LUTs and flip-flops. An alternative design is a linear feedback shift register counter (LFSR counter), which can be implemented very compactly using Xilinx's shift register LUTs (SRLs). For instance, a counter with period $2^{15}-1$ can be built from an SRL, a flip-flop, and a LUT configured as an XOR [Xilinx 2007]; this requires just 2 LUTs, compared to 15 for a binary counter. However, the count value is scrambled by the LFSR and must be recovered by solving the discrete logarithm problem [Clark and Weng 1994]. The discrete log is normally difficult to solve, which is why it has uses in the field of cryptography. LFSRs have been used as event counters in cases where slow offline decoding is acceptable, such as in silicon debug. The required overhead can be reduced somewhat through the choice of the LFSR's characteristic polynomial, but remains nontrivial. A series of exponentiations must be performed (via polynomial multiplication), followed by lookups to residue tables that must be held in memory, followed by an application of the Chinese remainder theorem

$$
\text { count }=\sum_{i=1}^{k} r_{i}\left(\frac{M}{m_{i}}\right) v_{i} \bmod M,
$$

where $k$ is the number of moduli, $r_{i}$ is a residue, $M$ is the counting period, $m_{i}$ is a modulus, and $v_{i}$ is a weight found with the extended Euclidean algorithm.

We propose a counter design that is both compact and relatively easy to decode: a residue number system (RNS) ring counter. An example is shown in Figure 2. This 


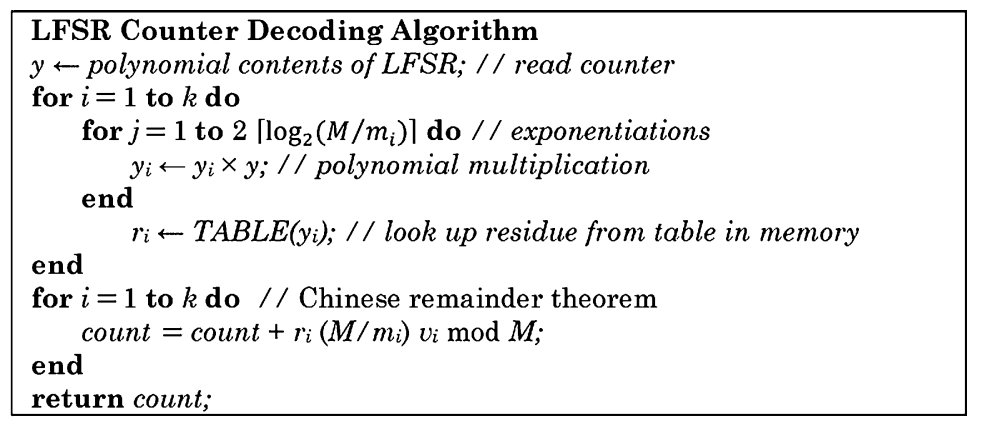

(a)

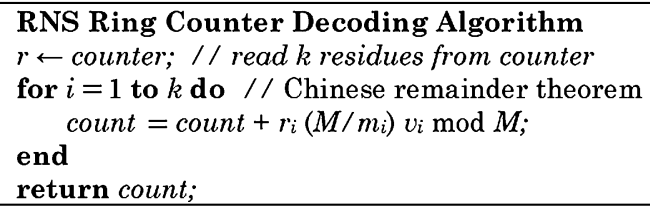

Fig. 3. Sketch of algorithms to recover the count for (a) an LFSR counter [Clark and Weng 1994] and (b) the proposed RNS ring counter.

Table I. Comparison of Counter Implementations Assuming a Maximum Period of $2^{13}$

\begin{tabular}{|c|c|c|c|}
\hline \multirow[b]{2}{*}{ Counter type } & \multicolumn{2}{|c|}{ Hardware overhead } & \multirow[b]{2}{*}{ Ease of decoding } \\
\hline & $\begin{array}{l}\text { Logic } \\
\text { (LUTs) }\end{array}$ & $\begin{array}{c}\text { Memory } \\
\text { (Bytes) }\end{array}$ & \\
\hline Binary & 13 & 0 & Trivial \\
\hline LFSR using SRLs & 2 & $\begin{array}{l}\text { Hundreds } \\
\text { [Clark\&Weng 1994] }\end{array}$ & $\begin{array}{l}\text { Dozens of polynomial multiplications; } \\
\geq 2 \text { table lookups; } \geq 4 \text { integer ops }\end{array}$ \\
\hline $\begin{array}{l}\text { RNS ring counter } \\
\text { using SRLs (proposed) }\end{array}$ & 2 & 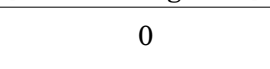 & 6 integer operations \\
\hline
\end{tabular}

style of counter was proposed long ago for generating timing signals [Fletcher et al. 1975], but to our knowledge its potential as an extremely efficient event counter for FPGAs has not been recognized. The design is composed of multiple shift registers of varying lengths, each feeding back to itself. Each shift register acts as a modulo- $m_{i}$ "ring counter" and can be implemented with an SRL plus an optional flip-flop. The shift registers are initialized with a pattern such as a one-hot codeword. When the count is to be incremented the patterns shift to the right; the position of each "hot" bit represents the count value modulo $m_{i}$, in other words, a residue $r_{i}$. A modulo-3 ring simply cycles through the states of 100, 010, and 001. By implementing rings whose moduli are all pairwise relatively prime, the counter can reach a period of $M=\prod m_{i}$. Since the residue values are readily available simply by reading out the individual shift registers, the RNS count value can be easily recovered just by applying the Chinese remainder theorem (3.1). Most of the processing overhead of an LFSR counter is avoided, and no memory is required for tables. Pseudocode for the two counter styles is shown in Figure 3.

Consider an example in which a counter of period $2^{13}$ is required. A comparison of the three implementation styles is shown in Table I. An RNS ring counter implementation needs just two LUTs. For instance, with the Virtex-5/6, one LUT can act as a 32-bit SRL and the other as two 16-bit SRLs. With three rings the period reaches 
$33 \times 17 \times 16=8976$, which is not as high as a two-LUT LFSR but meets the target of $2^{13}$. RNS designs are far more compact than a binary counter, and in this case just as small as the smallest LFSR counter. Moreover, the RNS implementation requires no tables in memory, while the LFSR counter requires multiple tables totaling on the order of hundreds of bytes. Lastly, the RNS ring counter is almost as trivial to decode as a binary counter, comparing favorably to the dozens of polynomial multiplications needed for an LFSR counter.

\subsection{Frequency Estimation}

By counting ring oscillator pulses, the average frequency of a ring oscillator $\mathrm{RO}_{i}$ at location $(x, y)$ over the sample period $t$ can be estimated using the rate of pulses during the period plus the average truncation error:

$$
\text { frequency }=\frac{\text { pulse count }+ \text { truncation error }}{\text { sample period }} .
$$

The truncation error is associated with the partial clock pulse at the end of the sample period. (If the ring oscillator was already running when the sample period began, there will be a partial pulse at the beginning as well.) By including the truncation term, the maximum quantization error is cut in half, for instance from 1 least-significant bit (LSB) to 0.5 LSB. Over a set $A$ of frequency samples, which may be distributed spatially and/or temporally, the variation can be expressed as follows:

$$
\text { coefficient of variation }(A)=\frac{\sigma_{A}}{\mu_{A}} \text {. }
$$

When the samples are from the same oscillator, the variation is caused by changes in the environmental conditions, random measurement error, and quantization error. The resolution of the frequency measurement is dictated by the average ring oscillator frequency and the length of the sample period:

$$
\text { resolution }=\frac{1}{\text { frequency } \times \text { sample period }} .
$$

For instance, a frequency of $250 \mathrm{MHz}$ and a sample period of $40 \mu \mathrm{s}$ allow a resolution of 1 part in 10,000 , or $0.01 \%$.

\subsection{Ring Oscillator Design}

Ring oscillators consist of a ring of elements with an odd number of inversions and an on/off control switch. For online sensing, the main requirements for a ring oscillator include good sensitivity to relevant physical parameters, ease of sampling quickly and accurately, and compactness. We propose two methods for better achieving these objectives: 1) opportunistic use of configurable logic, and 2) reducing the number of stages.

To improve ring oscillator sensitivity to physical parameters, we propose increasing the fraction of ring oscillator path delay that is due to transistors rather than wires. Since the effect of temperature, for instance, is normally stronger on transistor delay than on wire delay, this increases the overall sensitivity to temperature. A specific technique is to instantiate a latch along with each LUT. The latches can be held in the open state and can act as additional transistor delay. Latches are generally available near the LUTs and do not require significant additional wiring. A hardware experiment comparing ring oscillators both with and without latches will be described in Section 5 . Other researchers have used latches in a ring oscillator [Kohlbrenner and Gaj 2004], but the impact of latches on parameter sensitivity appears not to have been recognized. 


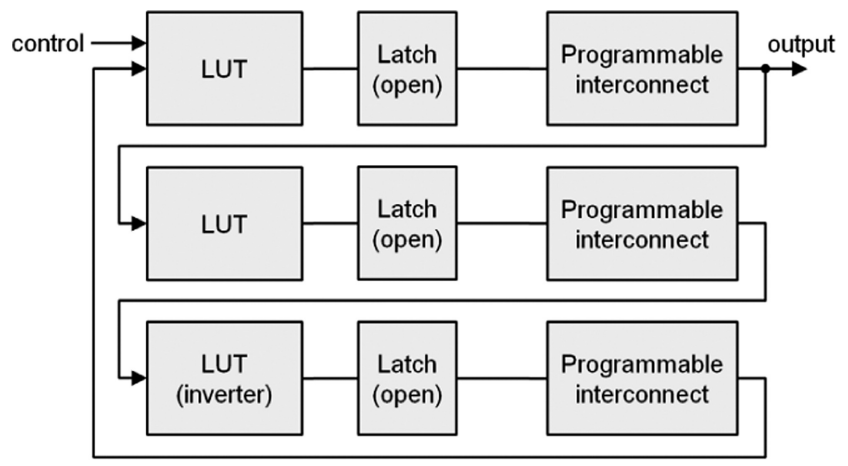

Fig. 4. Ring oscillator design.

In designing a ring oscillator, another degree of freedom is the number of stages $n$. Various numbers have been used in FPGA-based ring oscillators, including 4 [Boemo and López-Buedo 1997], 48 [Jones et al. 2007], and 75 [Chen et al. 2007]. The role of the number of stages has sometimes been misunderstood or overstated. First, consider random errors that are present in the oscillator readings. Oscillators with a higher number of stages will operate at lower frequencies. Thus the random measurement errors will naturally be much lower in absolute terms $(\mathrm{MHz})$. Some have taken this to mean that accuracy improves as $1 / n$ and that longer oscillators are strongly preferred (Figure 6 in Franco et al. [2010]). In fact, for an oscillator to be useful, the error must be small relative to the signal (i.e., frequency) being measured. The relative size of random measurement error does not improve linearly simply by adding stages. We demonstrate this empirically in Section 5 by comparing oscillators with 3 and 21 stages.

There is a separate rationale for using many stages in an oscillator design: local random component variation. The coefficient of variation across different oscillator instances due to this variation is related to the number of stages by [Takahashi et al. 2009]:

$$
\frac{\sigma_{\text {random component variation }}}{\mu_{\text {all instances }}} \propto \frac{1}{\sqrt{n}} .
$$

Oscillators with large $n$ will have less variability across instances, which may help in applications that rely on uncalibrated sensor readings [Sun et al. 2011]. However, with modern technologies, different oscillator instances can still be skewed by spatiallycorrelated variations, and thus calibration will in many cases still be required. If calibration is performed, then the local random variation across different oscillator instances is much less of a problem, and this particular rationale for large $n$ is less compelling. Given the above observations, greater consideration needs to be given to compact designs with few stages.

A basic ring oscillator design using our proposed latches and a small number of stages is shown in Figure 4.

\subsection{Reconfigurability}

It is highly desirable that sensor nodes support multiple modes of operation. However, in an ultra-compact design there may be no unused state elements available [Zick and Hayes 2010a]. We propose a simple solution for adding configurability at very low cost. One of the shift register rings can be used not only for counting but also to encode a configuration. For instance, in normal operation, the 17-bit shift register ring can be given a one-hot encoding. To signify a unique mode of operation, the shift register can be loaded instead with a different pattern. Only two bits of the ring are observable by 


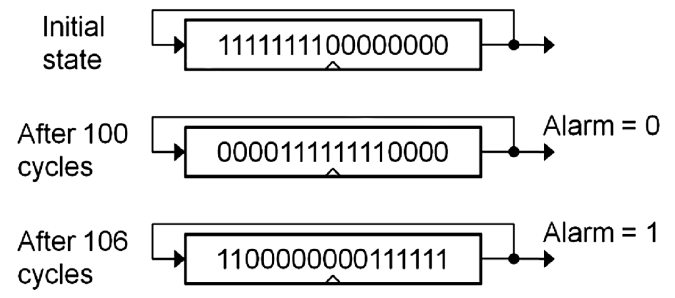

Fig. 5. Example of a 16-bit shift register ring used to distinguish between a valid sample count (assumed to be in the range of $100 \pm 3 \%$ ), and an invalid count (106) that is at or above a threshold (104).

logic: the output of the 16-bit SRL, and the output of the flip-flop. By simply ANDing those two bits, a mode select signal is generated with very little extra resources.

Adding Control of Individual Oscillators. In our previous design [Zick and Hayes 2010a], all ring oscillators were active during sampling. This allowed the control mechanism to be very simple, based on a pulse width modulation (PWM) timer signal distributed to all oscillators. However, in some cases only a subset of oscillators needs to be sampled. Thus it would be desirable if each oscillator could be individually configured to turn on or stay off during the PWM assertion. Keeping unused oscillators disabled would reduce parasitic interactions whereby oscillators are affected by one another, and would enable a slight reduction in dynamic power.

Controlling each oscillator with a unique, globally routed timer signal would cause congestion and is not scalable to the dense sensor arrays envisioned. Alternatively, a state element could be added to each sensor node and connected to the scan chain; the bit would configure the oscillator in enable or disable mode. However, no state elements are available in the ultra-compact design. We propose using a shift register ring pattern as mentioned above in order to generate the needed mode select signal.

\subsection{Low-Cost Threshold Sensing}

When combining online sensing with real-time control, there is a need for quickly determining whether an oscillator frequency is within a desired range. A frequency above or below a threshold can indicate a voltage emergency or other problem that needs to be detected immediately. An alarm signal needs to be generated for use in control logic in the region of the sensor or for generating a processor interrupt. Implementing this type of thresholding typically requires two registers in every sensor node to hold upper and lower threshold values, and the use of either dedicated comparator hardware or software processing. The Xilinx System Monitor includes registers and comparison logic for this purpose. For systems with many sensor nodes embedded within application logic, it would be beneficial to have a lower cost solution.

The value inside the proposed ring counter is encoded using the residue number system and is not in a form that can be readily compared to threshold values. One possibility would be using RNS arithmetic circuits, but this would not achieve the low overhead needed. A second problem is that the patterns inside the shift register LUTs are not observable, other than a single output bit. We propose using the ring counter in an elegant way. Instead of using a one-hot pattern, the longest shift register can be configured with a special pattern that acts as a model of two (or three) frequency bins. For instance, 0 bits can represent valid frequencies, while 1 bits indicate frequencies outside of one or more thresholds. After a frequency sample is taken, the shift register output directly represents whether the sampled count was in the valid range or not. A visualization of the concept is provided in Figure 5.

Note that an RNS ring counter used in this manner serves a dual purpose. The longest ring (modulus $m_{\max }$ ) provides the thresholding bit immediately after each sample, and 
at the same time represents the total count modulo $m_{\max }$, such that the exact count can still be eventually decoded as described in Section 3.1.

The sensor nodes need to be configured appropriately to enact this thresholding feature. The simplest case is when there is a single threshold. In that case, the range of expected counts can be no more than the largest modulus:

$$
\text { range of sample counts } \leq \max \left(m_{1}, m_{2}, \ldots m_{n}\right)=m_{\max } \text {. }
$$

The achievable resolution in the threshold values is a function of the modulus used and the range of frequencies that are to be supported (e.g., $+/-4 \%$ ):

$$
\text { resolution per count }=\frac{\text { frequency range }}{m_{\max }} \text {. }
$$

The pattern in the shift register ring needs to be initialized such that the fraction of "hot" bits reflects the relative size of the regions above and below the threshold:

$$
\text { hot bits in code word }=\left\lceil m_{\max } \times \frac{\text { threshold bin size }}{\text { frequency range }}\right\rceil \text {. }
$$

Moreover, the initial pattern should be offset within the shift register ring such that the final pattern will be lined up with the output:

$$
\text { offset }=-\left(\left\lceil\frac{m_{\max }}{\text { frequency range }}-\frac{m_{\max }}{2}\right\rceil \bmod m_{\max }\right) .
$$

As an example, if the range of expected sample cycles is 600 to 631 and the modulus is 32 , the initial offset would be $-(600 \bmod 32)=-24$. The maximum allowed sampling period (i.e., the length of time the oscillators are sampled) is:

$$
\text { sampling period } \leq\left\lfloor\frac{m_{\max }}{\text { frequency range }}\right\rfloor \times(\text { nominal oscillator period }) .
$$

Implementing two thresholds (3 bins) can be done similarly. The alarm signal indicates whether the frequency is valid or invalid; the actual frequency bin is found through post-processing. The hot bits associated with an upper threshold actually merge together with those associated with the lower threshold due to the way the shift register wraps around; this fact can be leveraged to increase the sampling time even further and thereby to improve the resolution.

\subsection{Sensor Node Design and Deployment}

The proposed sensor node design includes a ring oscillator, a frequency counter, and logic for control and access. A high-level diagram is shown in Figure 6. The sensor is enabled by a reference timer that is either on-chip or off-chip. A single timer can be used to enable multiple sensors simultaneously.

After a measurement, the sensor data is read out via a daisy chain. An array of sensors can be connected via one or more chains. The routing resources required include wires to daisy-chain the sensor nodes, a global timer signal, and one or more global signals for scan enabling/clocking. Embedded software controls the scan sequence and reads the sensor data. Note that the sensor data can be readily accessed without using the FPGA's built-in capability for reading out its configuration (readback), and similarly sensors can be initialized without involving the FPGA reconfiguration process.

The sensor node design can be defined with a schematic or an HDL netlist. Sensor locations can be easily specified by using physical constraint statements such as Xilinx's RLOC_ORIGIN. Directed routing statements are used to ensure consistent ring oscillator instances. Software drivers contain the code for operating the sensors 


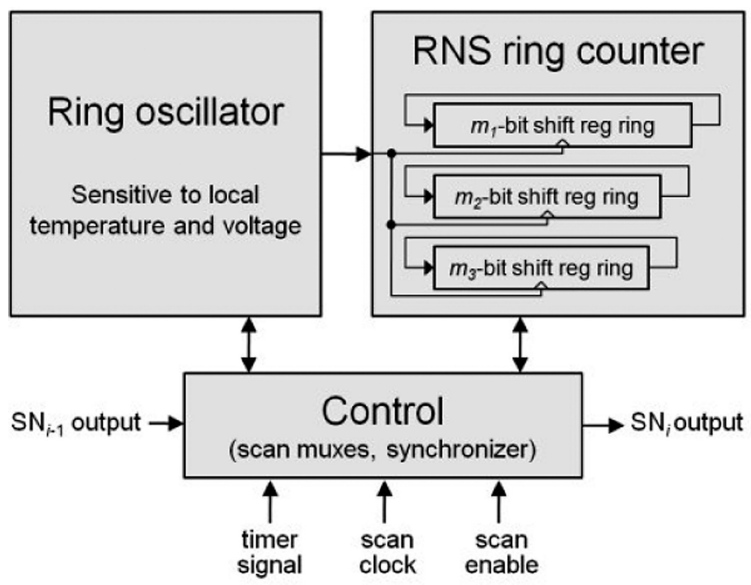

Fig. 6. High-level diagram of proposed sensor node.

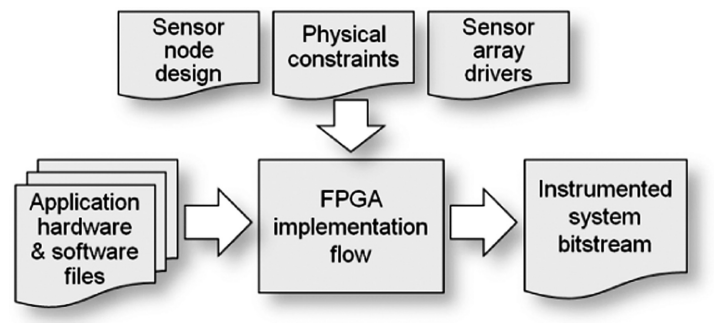

Fig. 7. Overview of the proposed design flow for FPGA system instrumentation.

and for accessing, decoding, and processing the data. By integrating the sensing infrastructure into a traditional system design flow, as shown in Figure 7, an instrumented application can be generated.

An interesting question is: what is an appropriate spatial arrangement of sensors? Related works involving FPGA-based thermal sensors have either suggested a regular rectangular grid, or an irregular arrangement focused on expected application-specific hotspots. Here we seek to perform systematic measurements of a variety of physical parameters, and thus employ a regular grid; specifically, we recommend a hexagonal tessellation due to its efficient area coverage [Wu and Verma 2008].

A second question is: what is an appropriate density of sensors? In the past, this has been largely dictated by hardware overhead. Most work on FPGAs involves between 1 and 32 sensors per chip. One work for ICs suggests sensing at 96 locations [Krishnamoorthy and Detofsky 2007]. Ideally, the sensor density should be high enough to match the finest spatial granularity of the physical phenomena being sensed. Higher sensor densities can enable increased accuracy, for instance, in predicting chip lifetimes [Sylvester et al. 2006]. To determine an appropriate density, one could use the distances at which parameter variations are correlated ("correlation distances"), and knowledge of the size of the system platform (e.g., die size). Unfortunately, with FPGA-based systems such information is not widely available. For instance, the correlation distance for supply voltage is not well understood, and the details of the power distribution system are proprietary. Given the heterogeneous nature of FPGA platforms, it is clear that there can be discontinuities (e.g., at boundaries of clock regions and of power regions) that prevent large correlation distances. Fortunately, with the proposed approach of 
ultra-compact sensor nodes, there is the possibility of extremely high sensor densities with little overhead. In principle, over 12,000 of the proposed sensor nodes could be instantiated in a high capacity Virtex-5 while using only half of the available slices. In practice, in order to keep the sensor node overhead near $1 \%$ on a Virtex-5, one can deploy slightly more than 100 sensor nodes.

\section{ON-LINE MEASUREMENT}

In this section we address three general questions: which physical parameters can be measured in a reconfigurable fabric, how is such data useful, and what are the procedures for measurement? We introduce enhanced procedures for measuring variations in four key parameters: delay, temperature, IR drop caused by switching activity, and IR drop caused by leakage current.

\subsection{Delay}

Every chip has fast areas and slow areas, due to factors such as imperfect lithography and variations in transistor threshold voltage or critical dimensions [Sedcole and Cheung 2006]. By mapping and adapting to delay variations, the system frequency can potentially be improved. Currently, the expected benefit is on the order of $10 \%$ [Srinivasan and Narayanan 2006; Cheng et al. 2006], and rising.

Spatially correlated delay variations can be mapped via controlled measurements of ring oscillator frequencies across a chip. A traditional procedure is to configure an FPGA fabric with a special test circuit containing ring oscillators. The chip is left in an idle state for an extended period (seconds to minutes) to minimize voltage and temperature gradients. Then the ring oscillators are sampled, either one at a time or (when feasible) simultaneously. We propose using a traditional procedure but with a key difference: the sensors are embedded in the application logic and the sensing is performed in the field. This reduces the bottleneck that occurs with expensive testers at manufacturing time. Furthermore, with an instrumented application there may be no need for storing a separate test bitstream and for performing reconfigurations.

\subsection{Temperature}

Thermal hotspots lead to early wearout, lower operating frequencies, higher static power, and extra cooling costs. Online sensing of a thermal profile can account for physical realities (e.g., variations in leakage or packaging) and allow for enhanced mitigation schemes.

There have been many attempts at estimating temperature through the use of ring oscillators, based on the premise that frequencies are related to temperature. Two opposing factors are in play: carrier mobility and transistor threshold voltage, both of which are temperature dependent. These effects are often expressed as:

$$
\begin{gathered}
\mu_{n}(T)=\mu_{n}\left(T_{0}\right)\left(\frac{T}{T_{0}}\right)^{\alpha_{\mu}}, \\
V_{T h}(T)=V_{T h}\left(T_{0}\right)+\alpha_{V T H}\left(T-T_{0}\right),
\end{gathered}
$$

where $T_{0}$ is a reference temperature and $\alpha_{\mu}$ and $\alpha_{V T H}$ are extracted parameters with negative values [Filanovsky and Allam 2001]. Traditionally, at higher temperatures, the carrier mobility decreases and acts to slow the logic switching speeds, while the threshold voltage decreases and acts to increase the switching speed. The conventional wisdom is that the net effect is a slowdown with temperature, where the relationship is both strong and linear. We will test in the next section whether these principles are still valid, and if so, to what extent. 
Many thermal sensing methods do not adequately address the problem of voltage noise. Local voltages cannot be easily controlled or measured, adding noise to temperature measurements. Previous works tend to assume that by pausing system activity, the supply voltage very quickly becomes spatially uniform and thus temperature can be cleanly estimated. This is no longer valid in general, since leakage variations (greatly amplified by temperature variations) cause nonuniform drops in the supply voltage. A second problem is that the supply voltage at the chip pins $\left(V C C_{p}\right)$ may not be measurable with fine resolution. For instance, the Xilinx System Monitor reports $V C C_{p}$ with only $3 \mathrm{mV}$ resolution, which could introduce errors of $10^{\circ} \mathrm{C}-20^{\circ} \mathrm{C}$ into temperature estimates. A third problem is that IR drop causes nonlinearity in the delay, temperature, and $V C C_{p}$ relationships, so the conventional linear models are becoming less accurate. Temperature sensing could be improved if additional builtin analog sensors were available on FPGAs, or if leakage current could be selectively disabled via power gating. Absent those, there is a need for new models and methods.

We propose the following procedure for estimating a thermal profile. First, ring oscillator measurements are made across the range of voltages and temperatures. A model of temperature as a function of delay and voltage is built, for instance via surface fitting in MATLAB. This characterization and modeling can be performed off-line and need only be done once for a given ring oscillator design and target platform type. An example is detailed in Section 5. Next, in-system calibration is performed at more than one temperature. Autonomous systems would need to either leverage natural swings in ambient temperature, control the die temperature via a fan, or temporarily raise the temperature profile using a reconfigurable "heater" circuit that consumes power appropriately. The calibration data is used to calculate the coefficients of the thermal model for each sensor. Finally, at runtime, the chip region being sensed must be paused and left idle for long enough to allow any voltage transients to dissipate. The ring oscillator frequencies are then measured, and the supply voltage at the pins is sampled (an average of multiple readings can improve the limited resolution). This data is then plugged into the empirical model and the local temperature is estimated.

\subsection{Switching-Induced IR Drop}

The switching activity in a system entails switching current and resulting IR drop. As mentioned earlier, IR drop leads to inefficiencies and potentially timing errors. While the spatial distribution of dynamic power consumption can usually be estimated at design time, or in some cases at runtime using performance counters, it is often not known how the dynamic power maps to IR drop. With FPGA-based systems, the power grid has irregularities and discontinuities [Lalgudi et al. 2005], and the details are proprietary, preventing accurate simulation. Moreover, with certain systems even the dynamic power profile may not be well understood, due to changes in workload or autonomously generated configurations. An ability to sense the switching-induced IR drop profile can enable better system health through adaptation (e.g., new system configurations) and better control policies.

We now describe a method of inferring the switching-induced IR drop profile. The basic approach is to sample ring oscillator frequencies during normal system operation, then briefly pause the activity of interest, and then immediately sample the frequencies again. The change in frequency represents the size of local IR drop due to switching current [Abuhamdeh et al. 2007; Wu et al. 2009]:

$$
\Delta V_{s w}(x, y)=K_{V} \times \Delta \operatorname{freq}(x, y) .
$$


The ratio of the frequency changes at two locations provides the ratio of the switchinginduced IR drops at those locations:

$$
\frac{\Delta V_{s w}(x 1, y 1)}{\Delta V_{s w}(x 2, y 2)}=\frac{K_{V} \times \Delta \text { freq }(x 1, y 1)}{K_{V} \times \Delta \text { freq }(x 2, y 2)}=\frac{\Delta \text { freq }(x 1, y 1)}{\Delta \text { freq }(x 2, y 2)} .
$$

Temperature and leakage effects remain nearly constant since the time between readings is much less than the thermal time constant which can be tens of milliseconds. The instrumentation itself (a timer and one or more sensor nodes) causes some switching activity, but with ultra-compact sensor nodes this activity is kept extremely low. Similar basic approaches have been used for estimating voltage variations [Conn 2000; Krishnamoorthy and Detofsky 2007]. We propose the following enhancement to previous methods. Voltage transients can introduce large errors in ring oscillator data. A simple check can be conducted for such events; instead of taking a single reading while the application is running we propose taking multiple consecutive readings. Each reading is spaced out by the length of the problematic transients (on the order of $1 \mathrm{~ms}$ ). A quick check of data consistency can determine whether a voltage transient event occurred. If not, the procedure can move forward and the switching activity can be paused; otherwise new readings are required. This method is most practical for applications with a relatively steady-state power profile or activity phases much longer than milliseconds. The proposed software-controlled procedure is as follows.

(1) Sample ring oscillator frequencies twice while application is running

(2) Check consistency; if a voltage transient is detected repeat 1

(3) Pause the switching activity

(4) Wait for voltage transients (caused by pausing activity) to dissipate

(5) Sample ring oscillator frequencies again

(6) Resume the switching activity

(7) When convenient, compare the frequency shifts of ring oscillators

\subsection{Leakage-Induced IR Drop}

Leakage is a phenomenon in which current flows through a transistor at the wrong time (i.e., when turned off) or wrong place (gate oxide), as with a leaky water faucet or pipe. The amount of variation is much larger than it is for delay; the ratio of leakage from worst-case to best-case transistors on a single FPGA die has already passed 2.0 [Wong et al. 2005]. Two main types are subthreshold leakage, which is very sensitive to temperature, and gate oxide leakage. With the high-K metal gate transistors used on 28nm FPGAs, gate oxide leakage may be largely mitigated [Betz 2010], meaning the relative importance of subthreshold leakage (and thus temperature) will increase. Hot transistors leak more current and generate more heat, driving a positive feedback loop. Leakage current leads to IR drop. It would be helpful to sense a chip's leakage-induced IR drop profile so the problems of static power, thermal hotspots, and temperaturedependent wearout could be more effectively mitigated. One recent work claims that variation-aware adaptation could reduce leakage by $14 \%$, even while simultaneously improving frequency [Srinivasan and Narayanan 2006]. With some FPGA platforms, leakage can be partially mitigated through fine-grained control of body bias. Currently, the body bias settings are chosen at presilicon time based on timing [Lewis et al. 2009]; with the ability to sense leakage variations online, systems will potentially be able to optimize their body bias settings online as well.

To our knowledge there has been no low-cost method of estimating a leakage-related profile in FPGAs. Srinivasan and Narayanan [2006] lament, "Programming leakage sensors on the FPGA may not be feasible to employ due to their analog nature." In a laboratory, one could carefully measure the leakage currents at various power supply 
pins and attempt to map the data to regions on the die, but this is not practical for production systems. As described in the previous section, isolating the effect of switching is straightforward; measurements can be taken both with and without the activity of interest while other variables are held nearly constant. The same is not usually true of leakage. Some chips do provide limited "power gating" in which the power to certain types of blocks can be completely shut off, but this is not available for the reconfigurable logic and interconnect.

We propose leveraging the fact that subthreshold leakage strongly depends on temperature:

$$
I_{\text {leak }}=k \times \mathrm{e}^{-\frac{q V t h}{a K_{b} T}},
$$

where $T$ is the absolute junction temperature, $V_{t h}$ is threshold voltage, $a$ and $k$ are device parameters, and $K_{b}$ and $q$ are physical constants [Kaxiras and Xekalakis 2004]. On modern chips using $65 \mathrm{~nm}$ and more advanced technology, this current is high enough to cause noticeable drops in the local supply voltages. Ring oscillator frequencies can be measured at two widely different temperatures while switching activity is minimized. The leakage currents will be higher in the hotter scenario and will cause additional IR drop which affects the ring oscillators. A complication is that other factors are at work: the oscillators will also be directly affected by the increase in temperature (Section 4.2), and by a temperature-dependent increase in resistance in the power grid, which increases IR drop [Ajami et al. 2003]. There are also interactions between these physical effects, such as the feedback between leakage and local temperature. Reiterating (2.1), the oscillator frequency can be measured empirically but in this case both the local voltage and local temperature are unknown:

$$
\text { frequency }(x, y)=f(V(x, y,), T(x, y)) \text {. }
$$

The local temperature depends on the local leakage, as does the local voltage, even if switching activity can be made negligible:

$$
V(x, y)=V d d-\left(\Delta V_{s w}+\Delta V_{\text {leak }}\right) \cong V d d-\Delta V_{\text {leak }} .
$$

The leakage-induced IR drop depends on the leakage current according to Ohm's Law, but also the power grid resistance, both of which depend in this case on the local temperature:

$$
\Delta V_{\text {leak }}(x, y, T)=I_{\text {leak }}(x, y, T) \times R(x, y, T) .
$$

Fortunately, we are not looking to characterize the size of the leakage variations in absolute terms, and thus need not untangle the different effects and their feedback paths. Rather, we aim to build a relative profile of a chip so that we know which regions have the most and least leakage-induced IR drop. Identifying outliers is important. For these goals, we note that the leakage-related effects all work in concert. Regions with highest leakage current will have slightly higher temperature and power grid resistance, and these will all tend to cause the largest slowdowns in a ring oscillator. Conversely, regions with the lowest leakage current will tend to have lower local temperature and resistance, and will tend to have the smallest slowdowns. The ordering of the chip regions by leakage-induced IR drop will be roughly preserved in the ordering of frequency shifts.

The measurement procedure involves performing ring oscillator delay characterization at disparate die temperatures, similar to the procedure mention in Section 4.2. With two or more widely-spaced measurements, the effects of increased temperature on each region of the chip can be compared, and the IR drop profile inferred.

As an example, imagine that ring oscillators spread across a die are sampled at stable, nearly-uniform temperatures of both $20^{\circ} \mathrm{C}$ (low leakage) and $85^{\circ} \mathrm{C}$ (high leakage). The 
Table II. Comparison of Reconfigurable-Logic-Based Sensors

\begin{tabular}{|l|c|c|}
\hline Sensor design & LUT count (target platform) & LUT count normalized to Virtex-5 \\
\hline [Chen et al. 2007] & 140 (Altera ACEX) & $\geq 70$ \\
\hline [Jones et al. 2007] & 100 (Virtex-4) & $\geq 50$ \\
\hline [López-Buedo et al. 2004] & 34 (Virtex-1) & 31 \\
\hline Design proposed here & 8 (Virtex-5) & 8 \\
\hline
\end{tabular}

Table III. Example Layout of Resources to Implement a Sensor Node in a CLB

\begin{tabular}{|c|c|l|}
\hline $\begin{array}{c}\text { Location } \\
\text { within slice }\end{array}$ & \multicolumn{1}{|c|}{ Slice 0 } & \multicolumn{1}{c|}{ Slice 1 } \\
\hline D & $\begin{array}{c}\text { 1b of SRL16 + D flip-flop to sync timer } \\
\text { signal; 16b of SRLC16 for 49b ring }\end{array}$ & LUT6 + latch for RO \\
\hline C & 32b of SRLC32E \& D flip-flop for 49b ring & Two LUT3 scan muxes for 49b \& 16b rings \\
\hline B & $\begin{array}{c}\text { LUT3 as scan mux + D flip-flop for 17b } \\
\text { ring; AND gate }\end{array}$ & LUT1 + latch for RO \\
\hline A & $\begin{array}{c}\text { 15b of SRL16 + D flip-flop for 16b ring; } \\
\text { 16b of SRLC16 for 17b ring }\end{array}$ & LUT1 + latch for RO; output mux \\
\hline
\end{tabular}

Table IV. Sensor Specifications

\begin{tabular}{|l|l|}
\hline Item & Specification \\
\hline Hardware resources & 8 LUTs $($ Virtex-5) \\
\hline Data size & 82 bits $(49+17+16)$ \\
\hline Resolution & 1 part in 10,000 assuming $40 \mu$ s measurement period \\
\hline Sensor array readout time & 82 bits $\times(\#$ of sensors $) \times($ scan frequency $)$ \\
\hline
\end{tabular}

difference in oscillator frequencies may turn out to be $2.0 \%$ on average due to global effects. However, one spatial region may exhibit larger differences of $2.5 \%$, and another close to 3\%. These spatial anomalies can provide indications of high leakage and IR drop.

\section{EXPERIMENTAL RESULTS}

We present the characteristics and overhead of the proposed sensing methods, and show the results of experiments involving delay, temperature, and two types of IR drop.

\subsection{Sensor Node Characteristics}

A distinguishing feature of the proposed sensor node is its compactness. The entire design fits into a single Virtex-5 CLB. This includes logic for scan and for synchronizing the timer signal to the ring oscillator clock. As can be seen in Table II, this is much smaller than previous designs that have been proposed for thermal sensing, even after normalizing to a single architecture. An example layout of resources within a CLB is shown in Table III. Key sensor specifications are shown in Table IV.

The ability of the sensor node to be used for thresholding can be calculated using equations (3.6)-(3.10). As a quantitative example, we assume that the size of the expected frequency range is $6 \%$ (in other words, $\pm 3 \%$ about the mean), and that the desired threshold is $-2 \%$ below the mean. The range of sample counts is 49 , while the resolution is $6 \% / 49=0.12 \%$. The number of "hot" bits needed in the code word is $\lceil 49 \times 1 \% / 6 \%\rceil=9$. The sampling period is $\lfloor 49 / 6 \%\rfloor \times 4 \mathrm{~ns}=3.26 \mu \mathrm{s}$. The initial offset for the code word in the shift register is $-(\lceil 49 / 6 \%-49 / 2\rceil \bmod 49)=-8$, and the initial value of the 49 -bit shift register ring is $0 \times 0000000000001 F F 00$. After each sample period, the output of the counter will indicate whether the frequency was above or below the threshold, as shown in Table V. 
Table V. Thresholding Example

\begin{tabular}{|c|c|c|}
\hline Sampled cycle count & Frequency estimate vs. mean (\%) & mod-49 counter output \\
\hline 840 & 2.89 to $3.01 \%$ & 0 \\
$\bullet$ & $\mathbf{\bullet}$ & $\bullet$ \\
\hline$\bullet$ & $\bullet$ & 0 \\
\hline 801 & -1.91 to $-1.78 \%$ & 1 \\
$\bullet$ & -2.03 to $-1.91 \%$ & $\bullet$ \\
\hline$\dot{\bullet}$ & $\bullet$ & $\bullet$ \\
\hline
\end{tabular}

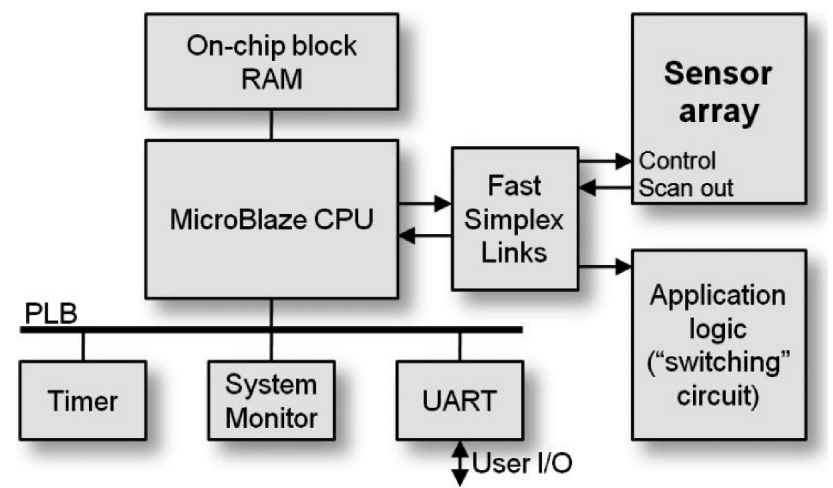

Fig. 8. Block diagram of the experimental system implemented on a Virtex-5 FPGA.

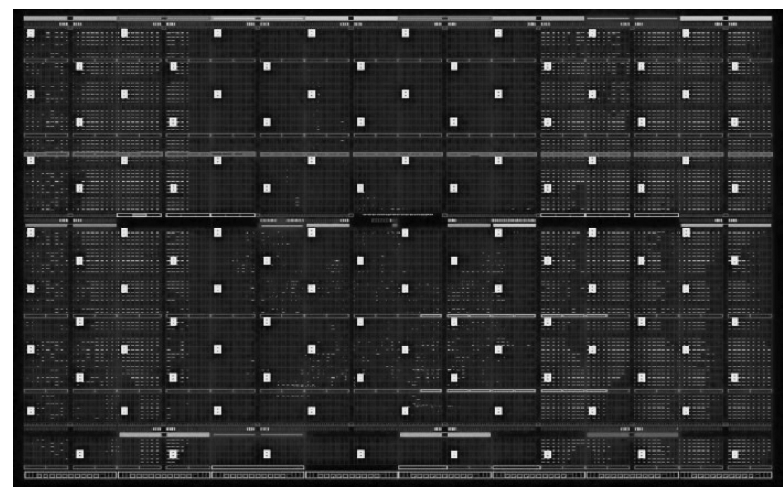

Fig. 9. Floorplan of an FPGA-based system instrumented with an array of compact multi-use sensors (light-colored rectangles) implemented in reconfigurable logic.

\subsection{Experimental System on a Chip}

We designed an experimental FPGA-based system and instrumented it with 112 sensor nodes. A block diagram of the design is shown in Figure 8. The design was instantiated on each of two Xilinx Virtex-5 FPGAs residing on XUPV5-LX110T boards. The reconfigurable fabric is composed of $160 \times 54$ CLBs, corresponding to $160 \times 108$ Virtex-5 slices. The sensors are arranged on a hexagonal grid of size $16 \times 7$ to fit the dimensions of the die. Sensors are nearly equidistant to each of their six neighbors. The layout can be seen in Figure 9, rotated right by 90 degrees. 

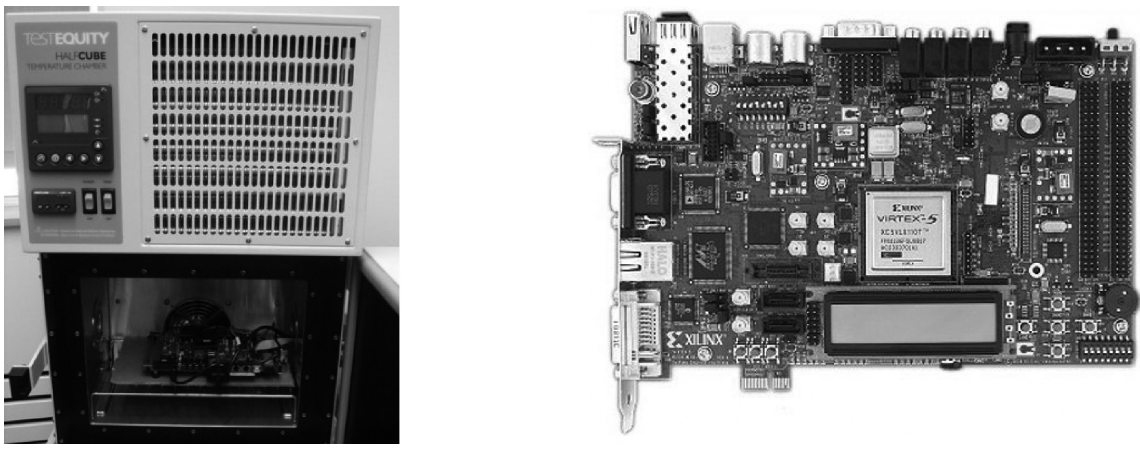

Fig. 10. Picture of the experimental setup in the thermal chamber (left). Picture of an XUPV5 circuit board (right).

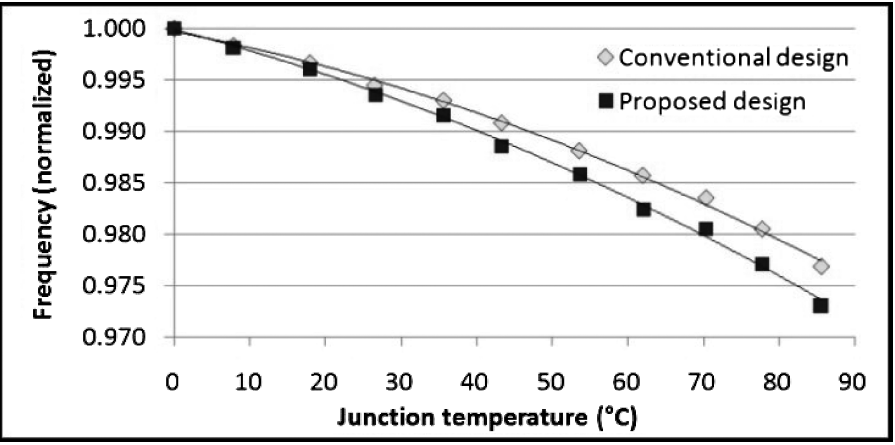

Fig. 11. Frequency dependence on temperature for proposed ring oscillator design and conventional design.

The system contains a MicroBlaze 7.10.a CPU and three peripherals connected to the processor local bus (PLB): an XPS Timer for enabling the sensors, a core for interfacing to the System Monitor, and a UART16550 for user input and output. All of these operate at $100 \mathrm{MHz}$. In addition, application logic was included for experimental purposes. The application logic acts as a switching/heater circuit with regions that can be independently enabled. It consists of 28,200 LUTs and flip-flops that can toggle at high speed. Both the array of sensors and the application logic are connected to the MicroBlaze via a shared set of fast simplex links of minimum size. The design was implemented with the Xilinx ISE and EDK 10.1 toolset. The experimental control software was written as a standalone application in $\mathrm{C}$ for the MicroBlaze, and fits into $32 \mathrm{~KB}$ of block RAM. Thermally-controlled experiments were performed using a TestEquity 105A thermal chamber, as pictured in Figure 10.

\subsection{Ring Oscillator}

We tested the proposed ring oscillator design that includes latches, and compared its temperature sensitivity to a four-stage conventional design without latches. Oscillator frequencies were measured at a range of temperatures from 0 to $85^{\circ} \mathrm{C}$, while the supply voltage at the pins was maintained at a constant $1.009 \mathrm{~V}$. The results demonstrate that the proposed design has a stronger temperature dependence than the conventional design, as seen in Figure 11. The relative improvement in the slope is $17 \%$.

Further experiments provide insight into random measurement error and the role of the number of oscillator stages. We collected sets of 10 measurements in quick succession and checked for consistency from each oscillator, using two instances of the 
Table VI. Comparison of Proposed Ring Oscillator Design with a 21-Stage Conventional Design

\begin{tabular}{|l|c|c|c|}
\hline Ring oscillator instance & $\begin{array}{c}\text { Average random } \\
\text { measurement error } \\
(\sigma / \mu) \text { across 8 sets } \\
\text { of 10 readings }\end{array}$ & $\begin{array}{c}\text { Average } \\
\text { frequency } \\
(\mathrm{MHz})\end{array}$ & $\begin{array}{c}\text { Resolution (normalized; } \\
\text { lower is better) }\end{array}$ \\
\hline Proposed 3-stage design, instance 1 & $0.0114 \%$ & 326.20 & 0.171 \\
\hline Proposed 3-stage design, instance 2 & $0.0094 \%$ & 319.70 & 0.174 \\
\hline 21-stage conventional design, instance 1 & $0.0053 \%$ & 53.54 & 1.000 \\
\hline 21-stage conventional design, instance 2 & $0.0078 \%$ & 55.74 & 0.961 \\
\hline
\end{tabular}

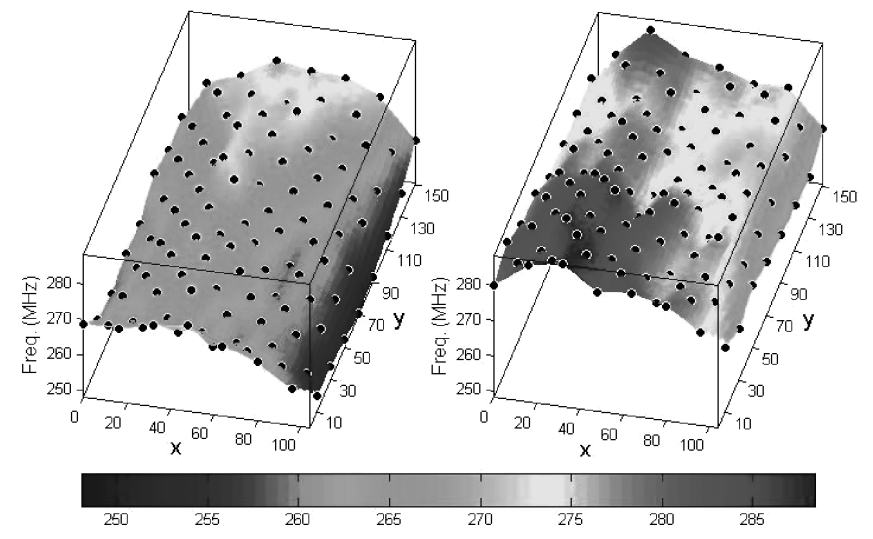

Fig. 12. Frequency profile for chip 1 (left) and chip 2 (right) in the idle state at $T_{j}=35^{\circ} \mathrm{C}$.

proposed design as well as two instances of a 21-stage, conventional design. The sample period was long enough $(1.33 \mathrm{~ms})$ to make quantization error negligible. The results are shown in Table VI. The random measurement error across each set of 10 measurements with the proposed design was quite low, roughly one part in $10,000(0.01 \%)$. This is nearly as low as for the 21-stage design, despite the extreme compactness. Furthermore, the proposed oscillator with its higher frequency enables roughly six times better frequency counter resolution for the same sample period, or a six times shorter sample period for the same resolution.

\subsection{Delay}

The first experiment using the proposed online measurement methods and full array of sensor nodes is a characterization of delay variations. This type of measurement is required for calibrating the ring oscillators. The ambient temperature was set to $25^{\circ} \mathrm{C}$ and the system was left in the idle state until it reached thermal equilibrium. The junction temperature was $35^{\circ} \mathrm{C}$ as reported by the System Monitor. Ten consecutive readings of the oscillators were taken. The frequency profiles of the two FPGA chips are shown in Figure 12. The $x$ and $y$ coordinates represent Virtex-5 slice locations. Dots indicate the average frequencies measured at the locations of the sensors. The frequencies at locations between sensors are estimated using linear interpolation. Several observations can be made. Chip 2 is noticeably faster than chip 1, due to inter-die variation and board-level differences (slightly different supply voltages and heat sinks). Here we are more concerned with intra-die variation. In both cases, the right side of the chip is the slowest region. The fastest region is near the top of the grid for chip 1 but near the bottom for chip 2. The distribution of measured frequencies is shown in Figure 13. The amount of spatial variation can be expressed via the coefficient of variation, which is the standard deviation $\sigma$ across all sensor frequencies divided by 


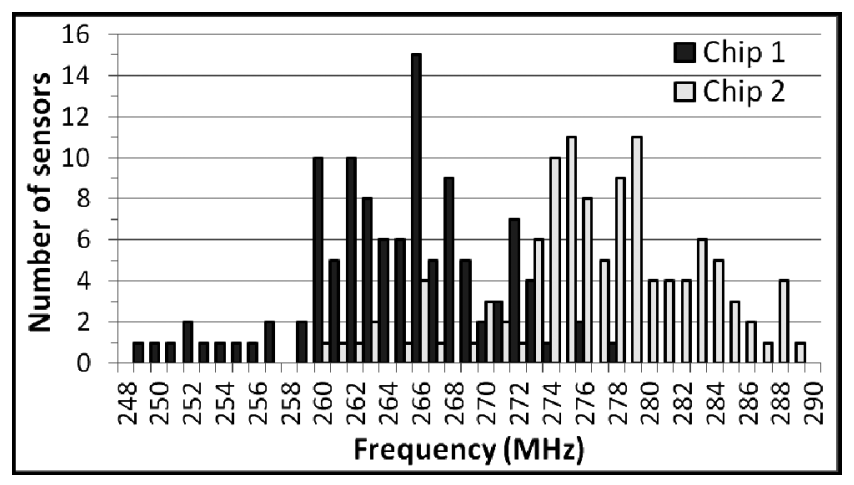

Fig. 13. Histogram of ring oscillator frequencies for chip 1 (dark) and chip 2 (light) in the idle state at $T_{j}=$ $35^{\circ} \mathrm{C}$.

the mean $\mu$. Both chips exhibit a coefficient of variation of approximately $2.3 \%$, which corresponds to a $3 \sigma$ variation of approximately $7 \%$.

\subsection{Temperature}

Next we consider temperature variations. We first build an empirical model of frequency, temperature and voltage. The sensor array is placed such that one sensor is immediately adjacent to the System Monitor at $(x, y)=(48,81)$, allowing temperature estimates for that location to be validated against the built-in analog sensor. We want to know what type of model fits the data and with what accuracy. We measured frequencies over a range of temperatures from 0 to $85^{\circ} \mathrm{C}$, and over a $40 \mathrm{mV}$ range of voltages. The supply voltage $V C C_{p}$ was indirectly controlled by enabling various amounts of application activity (affecting the switching-induced IR drop), from zero activity all the way to $100 \%$ application activity in $10 \%$ steps (equivalent to about 600 slices of application logic). In total, 34 data points were collected, with 10 readings at each point. Various models were fitted to the data using MATLAB, in order to find an effective temperature estimation function $T(x, y)=f\left(f r e q(x, y), V C C_{p}\right)$, where $T(x, y)$ is the temperature at die location $(x, y)$, and $f$ is a function of frequency freq $(x, y)$ and voltage $V C C_{p}$. We found that a traditional model with linear dependence on frequency and voltage provides a root mean square error of $5.7^{\circ} \mathrm{C}$. In contrast, we found that a $2^{\text {nd }}$ order polynomial model provides a better fit and lower error. The form of the model is as follows, with coefficients $c_{i}$ :

$$
T(x, y)=c_{1} \text { freq }(x, y)^{2}+c_{2} V C C_{p}^{2}+c_{3} \text { freq }(x, y) V C C_{p}+c_{4} f r e q(x, y)+c_{5} V C C_{p}+c_{6} .
$$

This model reduces the error to $3.5^{\circ} \mathrm{C}$. Plots of the surface fit can be seen in Figure 14; the surface is curved and twisted rather than planar. The error with this model is comparable to the accuracy of analog sensors such as the one built in to the System Monitor, which is rated to $\pm 4^{\circ} \mathrm{C}$.

In addition to the validated measurements at location $(48,81)$, we sensed the thermal effects across the entire fabric for the application we have mentioned. Ring oscillator frequencies were measured immediately after the application was paused, and compared to the steady-state idle case. The resulting shifts in frequencies are illustrated in Figure 15. This profile includes not only the direct effect of temperature, but also the indirect effect caused by temperature-dependent leakage.

We quantified the sensitivities of the two ring oscillator designs to temperature when implemented on Virtex-5, and compared the results to a compilation of empirical results previously published; see Table VII. The implications of these findings are discussed in Section 6. 


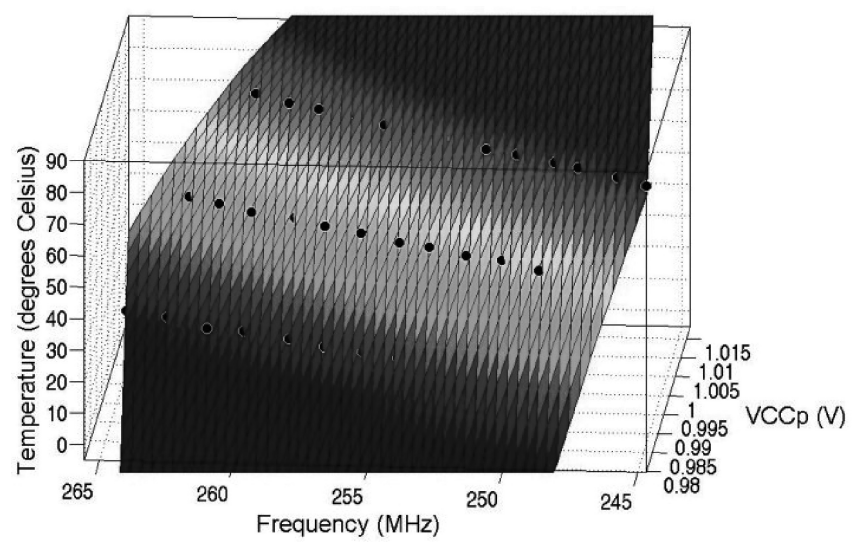

Surface fit using (5.1):

R-square: 0.9853

RMSE: 3.519

Coefficients:

$c_{1}=-38.15 ; c_{2}=-50.23$

$c_{3}=89.39 ; c_{4}=-102.7$;

$c_{5}=89.26 ; c_{6}=48.89$

where $\mathrm{x}$ is normalized by mean 255.8

and std 5.437 and where $\mathrm{y}$ is

normalized by mean 1.001 and std

0.01023

Fig. 14. Measured relationship between temperature, sensor frequency, and supply voltage for the sensor at $(48,81)$

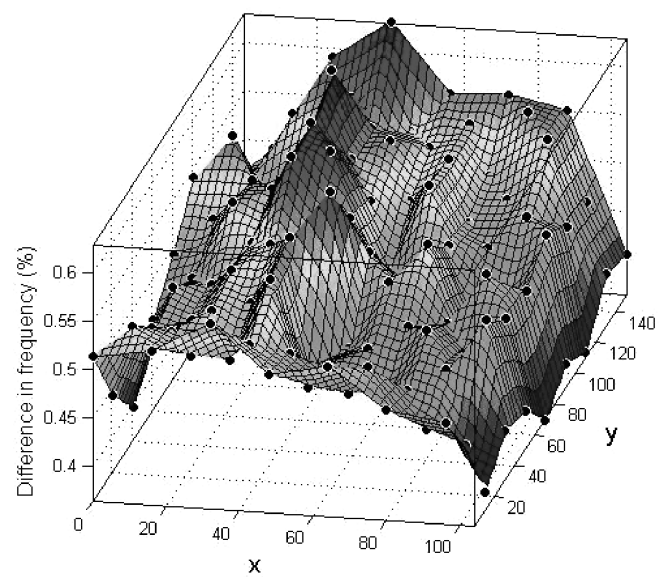

Fig. 15. Map of frequency changes due to temperature effects.

Table VII. Historical Data on FPGA Ring Oscillator Frequency Sensitivity to Temperature

\begin{tabular}{|l|l|c|c|c|}
\hline Study & \multicolumn{1}{|c|}{ Chip type } & $\begin{array}{c}\text { Process technology } \\
\text { (feature size, } \\
\text { core voltage) }\end{array}$ & $\begin{array}{c}\text { Temper. range } \\
\text { measured }\left({ }^{\circ} \mathrm{C}\right)\end{array}$ & $\begin{array}{c}\text { Frequency decrease } \\
\text { across measured } \\
\text { range (\%/ }{ }^{\circ} \text { C) }\end{array}$ \\
\hline [Xilinx 1997] & Xilinx XC3000 & $600+\mathrm{nm}, 5 \mathrm{~V}$ & -30 to 130 & 0.22 at $4.5 \mathrm{~V}$ \\
\hline [Boemo et al. 1997] & Xilinx XC3000 & $600+\mathrm{nm}, 5 \mathrm{~V}$ & $\sim 20$ to 100 & $0.30-0.35$ \\
\hline [López-Buedo et al. '97] & Xilinx XC4000 & $600+\mathrm{nm}, 5 \mathrm{~V}$ & 20 to 120 & $0.30-0.35$ \\
\hline [Chen et al. 2007] & Altera ACEX & $220 \mathrm{~nm}, 2.5 \mathrm{~V}$ & -40 to 130 & 0.12 \\
\hline [Sheldon et al. 2007] & Xilinx Virtex-II & $180 \mathrm{~nm}, 1.5 \mathrm{~V}$ & 50 to 125 & $0.14-0.15$ \\
\hline [Velusamy et al. 2004] & Xilinx Virtex-IIPro & $130 \mathrm{~nm}, 1.5 \mathrm{~V}$ & 35 to 60 & $0.19-0.21$ \\
\hline [Jones et al. 2007] & Xilinx Virtex-4 & $90 \mathrm{~nm}, 1.2 \mathrm{~V}$ & $\sim 34$ to 79 & 0.11 \\
\hline [Mangalagiri et al. '08] & Xilinx Virtex-5 & $65 \mathrm{~nm}, 1 \mathrm{~V}$ & 32 to 60 & $\sim 0.036$ \\
\hline This work & Xilinx Virtex-5 & $65 \mathrm{~nm}, 1 \mathrm{~V}$ & 0 to 85 & 0.032 (proposed RO); \\
& & & & 0.027 (standard RO) \\
\hline
\end{tabular}




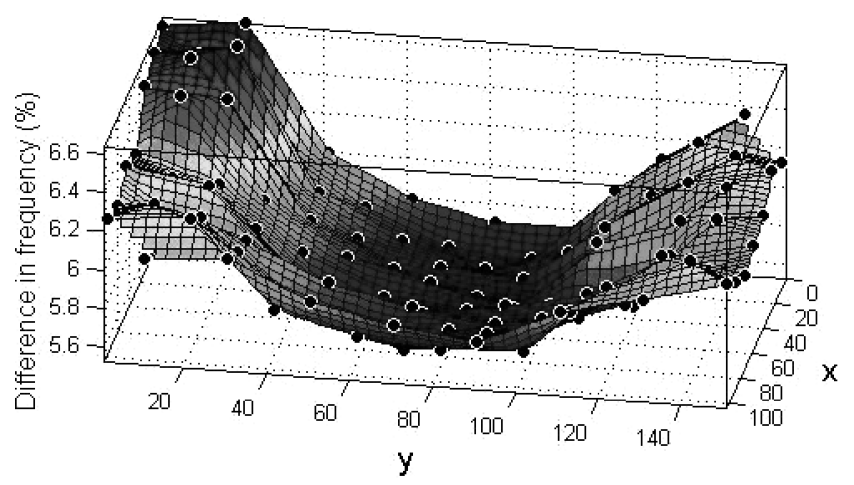

Fig. 16. Map of frequency changes due to switching-induced IR drop.

\subsection{Switching-Induced IR Drop}

A demonstration application was set up to generate high switching activity near the top and bottom of the reconfigurable fabric. The middle portion was left idle so that clean measurements could be taken and validated against the System Monitor, which sits near the center of the die. Specifically, 4,500 slices toggled in the upper third of the die with $y$ coordinates $\geq 110$, and 2,550 slices toggled in the bottom portion of the die. The proposed procedure was applied, isolating the effect of switching activity.

Measurements indicate that ring oscillator shifts are indeed largest in the two regions with switching activity, as shown in Figure 16. The image is rotated to illustrate the changes along the $y$ axis. This type of measurement provides important information about the spatial extent of switching effects. For instance, while the application was set up with a dramatic discontinuity in activity at $y=110$, the measured frequency shifts are surprisingly continuous, showing a relatively even slope that begins around $y=100$ and extends all the way to the top of the die.

This type of sensing can help quantify both global and local components of IR drop. There is a large global slowdown due to global IR drop; all oscillators slow by at least $5.6 \%$, even near the center of the die where negligible dynamic power is consumed. Superimposed upon this effect is an additional slowdown of up to $1 \%$ due to local IR drop in the regions with large switching activity.

\subsection{Leakage-Induced IR Drop}

First, we observed IR drop in the VCCINT power supply, using the built-in System Monitor analog voltage sensor which is connected to a chip power pin. We measured $V C C_{p}$ over the full range of supported junction temperatures while switching activity was minimized, and detected a decrease of several $\mathrm{mV}$ when comparing $0^{\circ} \mathrm{C}$ to $85^{\circ} \mathrm{C}$. The voltage difference was roughly $1 \mathrm{mV}$ from $75^{\circ} \mathrm{C}$ to $85^{\circ} \mathrm{C}$ on the Virtex-5 we tested.

We measured all ring oscillator frequencies at two disparate junction temperatures, $35^{\circ} \mathrm{C}$ and $85^{\circ} \mathrm{C}$, while switching activity was kept to a minimum. The ambient temperature was controlled by the thermal chamber. The resulting frequency shifts are visualized in Figure 17. Larger shifts indicate larger IR drop. Certain ring oscillators on chip 1 slow down by $1.6 \%$, while others slow down by $2.7 \%$. For chip 2 the range is $1.3 \%$ to $2.4 \%$.

\section{DISCUSSION}

The proposed approach and our findings have several implications for future system design and research. 


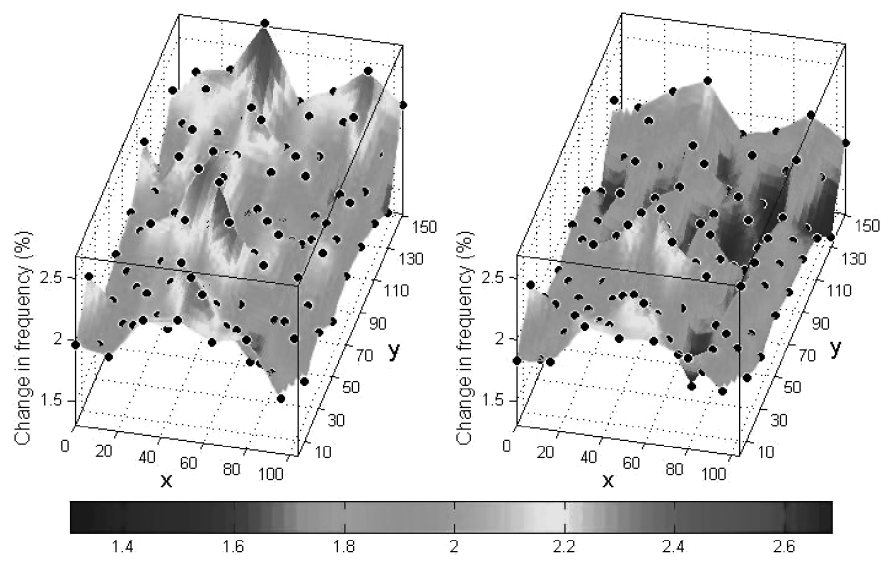

Fig. 17. Map of frequency shifts for chip 1 (left) and chip 2 (right), providing a sense of the leakage-induced IR drop profile.

Sensing temperature. Historically, the dependence of FPGA ring oscillator delay on temperature was strong. As seen in Table VII, with the Xilinx XC3000 chips in the mid-1990s, ring oscillator frequencies would decrease by anywhere from 0.20 to $0.35 \%$ per ${ }^{\circ} \mathrm{C}$ [Boemo and López-Buedo 1997]. Even with the Xilinx Virtex-4 FPGA, published data shows a strong dependence [Jones et al. 2007]. Our Virtex-5-based study finds that the temperature dependence is now very weak, and that the conventional wisdom no longer applies. When comparing the frequencies at highest vs. lowest temperature, our standard-style ring oscillator slows by a mere $0.027 \% /{ }^{\circ} \mathrm{C}$. With our addition of latches, the sensitivity increased somewhat to $0.032 \% /{ }^{\circ} \mathrm{C}$, but this is still an order of magnitude lower thermal response than in technologies past. These empirical results help to confirm and quantify the trend long predicted (for one example, see Filanovsky and Allam [2001]). With more advanced technologies, power supply voltages are lower and the carrier mobility effect is no longer as dominant. In fact, with upcoming technologies the nominal supply voltage will be near the point at which two effects balance each other and logic speeds are not affected by temperature at all. One study predicts that with $22 \mathrm{~nm}$ technologies, this zero-temperature dependence point will be reached at voltages of $0.73 \mathrm{~V}$ [Wolpert and Ampadu 2009]. Going forward, sensing temperature with standard reconfigurable logic running at the core voltage will be increasingly problematic, and will require extraordinarily quiet supply voltages. Fortunately, sensors of this type are very promising for parameters others than temperature.

Wearout. Characterization of delays is desirable not only for manufacturing process variations but for tracking gradual shifts caused by wearout. Wearout mechanisms include negative bias temperature instability, total ion dose, electromigration, and time-dependent dielectric breakdown. These can occur at various spatial and temporal scales, and can be accelerated by regional effects such as thermal hot spots. Further studies are needed regarding in-system sensing of wearout at the various spatial and temporal scales.

Much higher sample rates. There is a vast spectrum of voltage noise at high frequencies. In Joseph et al. [2003], the important frequencies for voltage transients are $50-100 \mathrm{MHz}$. Counting RO pulses takes too long to characterize effects this fine. A possible approach would be to indirectly measure the frequency of any ring oscillator using a separate, specialized circuit, such as certain types of time-to-digital converters able to measure short pulses with high precision and resolution [Aloisio et al. 2007]. The goal of this type of sensing would not necessarily be real-time control for voltage noise, but 
rather to uncover detailed information about the nature of the noise, to find systematic problems, and to enable system adaptation through methods such as re-placement.

Implications for ASIC- and MPU-based systems. The overall approach to online sensing and many of the specific methods have relevance not just for FPGA-based systems but also for ASIC- and MPU-based systems. Although ASICs and MPUs lack the finely reconfigurable logic of FPGAs, they often have enhanced configurability in other areas, such as dynamic voltage scaling, adaptive body bias, power gating, and many-core scheduling. Low-cost on-line sensing can help inform the control policies and adaptation algorithms.

Implications for future FPGA-based systems. As reconfigurable technology advances deeper into the nanoscale, the physical landscape is getting much more complex. New wearout mechanisms are emerging. Higher power densities are exacerbating IR drop. Operating conditions are becoming more dynamic; with $28 \mathrm{~nm}$ FPGAs, for the first time systems can employ dynamic voltage scaling and power gating of on-chip SRAMs [Xilinx 2011], leading to new sources of voltage transients. At the same time, voltage requirements are tightening, from $\pm 5 \%$ to a mere $\pm 3.3 \%$ ( $30 \mathrm{mV}$ at $0.9 \mathrm{~V})$. Multidie stacked silicon chips (“2.5D”) are arriving, with $3 \mathrm{D}$ looming. The dies within a stacked silicon chip can have significantly different physical characteristics such as leakage. Importantly, all of the above physical phenomena have the potential to interact with each other in new and unpredictable ways. Further progress on low-cost, fine-grained on-line sensing will be essential for reconfigurable systems to flourish.

\section{CONCLUSIONS}

This article introduced an approach to online sensing that includes compact multiuse sensor nodes and enhanced procedures for measuring physical parameters of key interest to system designers. Novel use of a residue number system counter yields sensors that are surprisingly compact, while an enhanced ring oscillator enables a $17 \% \mathrm{im}$ provement in temperature sensitivity. The proposed sensor node fits into just 8 LUTs on a Virtex-5, roughly four times smaller than the smallest previous design. Results indicate the proposed approach is suitable for low-cost sensing of a variety of parameters, and is accurate enough to be useful in a wide range of online adaptations. We see a growing need for this type of online sensing in support of physically-adaptive computing, with the goal of more efficient, more reliable, and healthier systems.

\section{ACKNOWLEDGMENTS}

We would like to thank Matthew French, Yiqian Min, Andrea Pellegrini, Joseph Greathouse, Robert Dick, USC ISI colleagues, and David Blaauw for assistance; and Xilinx Inc. and Sun Microsystems for equipment donations.

\section{REFERENCES}

Abuhamdeh, Z., Hannagan, B., Crouch, A. L., And Remmers, J. 2007. A production IR-drop screen on a chip. IEEE Des. Test Comput. 24, 3, 216-224.

Ajami, A. H., Banerjee, K, Mehrotra, A., And Pedram, M. 2003. Analysis of IR-drop scaling with implications for deep submicron PIG network designs. In Proceedings of the Symposium on Quality Electronic Design. $35-40$.

Aloisio, A, Branchini, P., Cicalese, R., Giordano, R., Izzo, V., and Loffredo, S. 2007. FPGA implementation of a high-resolution time-to-digital converter. In Nuclear Science Symposium Conference Record. 504-507.

Betz, V. 2010. FPGAs at 28nm: meeting the challenge of modern systems-on-a-chip. In Proceedings of the Conference on Field Programmable Logic and Applications.

Boemo, E. ANd López-Buedo, S. 1997. Thermal monitoring.on FPGAs using ring-oscillators. In Proceedings of the Workshop on Field-Programmable Logic and Applications. 69-78.

BongaRd, J., Zykov, V., AND Lipson, H. 2006. Resilient machines through continuous self-modeling. Science $314,5802,1118-1121$. 
Chen, P., Shie, M., Zheng, Z.-Y, Zheng, Z.-F., And Chu, C. 2007. A fully digital time-domain smart temperature sensor realized with 140 FPGA logic elements. IEEE Trans. Circ. Syst. 1, 54, 12, 2661-2668.

Cheng, L., Xiong, J., He, L., AND Hutton, M. 2006. FPGA performance optimization via chipwise placement considering process variations. In Proceedings of the Conference on Field Programmable Logic and Applications. 1-6.

Clark, D. And Weng, L. 1994. Maximal and near-maximal shift register sequences: efficient event counters and easy discrete logarithms. IEEE Trans. Comput. 43, 5, 560-568.

ConN JR., R. 2000. Method and apparatus for measuring localized temperatures on integrated circuits. U.S. Patent 6067508.

Dighe, S., VANGAL, S., ET AL. 2010. Within-die variation-aware dynamic-voltage-frequency scaling core mapping and thread hopping for an 80-core processor. In Proceedings of the International Conference on Solid-State Circuits. 174-175.

Filanovsky, L. M. AND Allam, A. 2001. Mutual compensation of mobility and threshold voltage temperature effects with applications in CMOS circuits. IEEE Trans. Circ. Syst. 148,7 , 876-884.

Flatley, T. 2010. Advanced hybrid on-board science data processor-SpaceCube 2.0. In Proceedings of the Earth Science Technology Forum.

Fletcher, J., Perlman, M., Rousey, W., And Messner, A 1975. System for generating timing and control signals. U.S. Patent 3866022.

Franco, J. J. L., Boemo, E., Castillo, E., and Parrilla, L. 2010. Ring oscillators as thermal sensors in FPGAs: Experiments in low voltage. In Proceedings of the Southern Programmable Logic Conference. 133-137.

International Technology Roadmap for SEMiconductors. 2011. http://www.itrs.net.

JiANG, N. AND PARASHAR, M. 2009. Enabling autonomic power-aware management of instrumented data centers. In Proceedings of the Symposium on Parallel \& Distributed Processing. 1-8.

Jones, P. H., Moscola, J., CHo, Y. H., AND Lockwood, J. W. 2007. Adaptive thermoregulation for applications on reconfigurable devices. In Proceedings of the Conference on Field-Programmable Logic and Applications. 246-253.

Joseph, R., Brooks, D., AND Martonosi, M. 2003. Control techniques to eliminate voltage emergencies in high performance processors. In Proceedings of the Symposium on High-Performance Computer Architecture. 79-90.

KaXiRAs, S. AND Xekalakis, P. 2004. 4T-decay sensors: a new class of small, fast, robust, and low-power, temperature/leakage sensors. In Proceedings of the Symposium on Low Power Electronics and Design. 108-113.

Kohlbrenner, P. And GaJ, K. 2004. An embedded true random number generator for FPGAs. In Proceedings of the Symposium on Field Programmable Gate Arrays. 71-78.

Krishnamoorthy, A. AND Detofsky, A. 2007. Mapping variations in local temperature and local power supply voltage that are present during operation of an integrated circuit. U.S. Patent 7233163.

Lalgudi, S. N., Kretchmer, Y. K., and Swaminathan, M. 2005. Simulation of switching noise in on-chip power distribution networks of FPGAs. In Proceedings of the Electrical Performance of Electronic Packaging. $319-322$.

Lewis, D. M., Ahmed, E., Cashman, D., Vanderhoek, T., Lane, C., Lee, A., and Pan, P. 2009. Architectural enhancements in Stratix-Ill and Stratix-IV. In Proceedings of the Symposium on Field-Programmable Gate Arrays. 33-42.

LóPEz-BuEdo, S. AND BoEmo, E. 1997. A method for temperature measurement on reconfigurable systems. In Proceedings of the Conference on Design of Circuit and Integrated Systems. 727-730.

LóPEz-Buedo, S. ANd Boemo, E. 2004. Making visible the thermal behavior of embedded microprocessors on FPGAs: A progress report. In Proceedings of the Symposium on Field Programmable Gate Arrays. 79-86.

Mangalagiri, P., Bae, S., Krishnan, R., Xie, Y., and Narayanan, V. 2008. Thermal-aware reliability analysis for platform FPGAs. In Proceedings of the Conference on Computer Aided Design. 722-727.

Muhtaroglu, A., Taylor, G., and Rahal-Arabi, T. 2004. On-die droop detector for analog sensing of power supply noise. IEEE J. Solid-State Circ. 39, 4, 651-660.

Quénot, G. M., PARIS, N., AND Zavidovique, B. 1991. A temperature and voltage measurement cell for VLSI circuits. In Proceedings of the European ASIC. 334-338.

Sedcole, P. And Cheung, P. 2006. Within-die delay variability in 90nm FPGAs and beyond. In Proceedings of the Conference on Field Programmable Technology. 97-104.

Sheldon, D., Roosta, R., SAdigursky, M., and Farrokhy, A. 2007. Monitoring temperature in SRAM-based FPGAs using a ring-oscillator design. In Proceedings of the Military and Aerospace FPGA and Applications Meeting. 
SRinivasan, S. And Narayanan, V. 2006. Variation aware placement for FPGAs. In Proceedings of the Conference on Emerging VLSI Technology and Architectures.

Sun, J., Bittner, R., And Eguro, K. 2011. FPGA side-channel receivers. In Proceedings of the Symposium on Field-Programmable Gate Arrays. 267-276.

Sylvester, D., BlaAuw, D., ANd KarL, E. 2006. ElastIC: An adaptive self-healing architecture for unpredictable silicon. IEEE Des. Test Comput. 23, 6, 484-490.

Takahashi, T., Uezono, T., Shintani, M., Masu, K., and Sato, T. 2009. On-die parameter extraction from pathdelay measurements. In Proceedings of the IEEE Asian Conference on Solid-State Circuits. 101-104.

VAhID, F., StitT, G., AND Lysecky, R. 2008. Warp processing: dynamic translation of binaries to FPGA circuits. IEEE Comput. 41, 7, 40-46.

Velusamy, S., Huang, W., Lach, J., and Skadron, K. 2004. Monitoring temperature in FPGA based SoCs. CS Tech. rep. CS-2004-39, University of Virginia.

WolPERT, D. AND AMPADU, P. 2009. A sensor to detect normal or reverse temperature dependence in nanoscale CMOS circuits. In Proceedings of the IEEE Symposium on Defect and Fault Tolerance in VLSI Systems. 193-201.

Wong, H. Y., Cheng, L., Lin, Y., ANd He, L. 2005. FPGA device and architecture evaluation considering process variations. In Proceedings of the Conference on Computer-Aided Design. 19-24.

Wu, C. AND Verma, D. 2008. A sensor placement algorithm for redundant covering based on Riesz energy minimization. In Proceedings of the Symposium on Circuits and Systems. 2074-2077.

Wu, T.-Y., Gharahi, S., And Aвraham, J. A. 2009. An area efficient on-chip static IR drop detector/evaluator. In Proceedings of the Symposium on Circuits and Systems. 2009-2012.

XILINX Inc. 1997. XC3000 series technical information. XAPP 024 (v 1.0).

XILINX INC. 2007. Linear feedback shift registers in virtex devices. App. note XAPP210 (v1.3).

XILINx Inc. 2009. Xilinx UG 192 Virtex-5 FPGA System Monitor User Guide, v1. 7.

XILINX INC. 2010. Radiation-hardened, space-grade Virtex-5QV device overview. DS192 (v1.1).

XILINX Inc. 2011. Lowering power at $28 \mathrm{~nm}$ with Xilinx 7 series FPGAs. WP389 (v1.0).

ZICK, K. M. AND HaYES, J. P. 2010a. On-line sensing for healthier FPGA systems. In Proceedings of the Symposium on Field-Programmable Gate Arrays. 239-248.

Zick, K. M. And Hayes, J. P. 2010b. Self-test and adaptation for random variations in reliability. In Proceedings of the Conference on Field Programmable Logic and Applications. 193-198.

Zick, K. M. AND HaYes, J. P. 2010c. Toward physically-adaptive computing. In Proceedings of the IEEE Conference on Self-Adaptive and Self-Organizing Systems. 124-133.

Received April 2011; revised July 2011; accepted September 2011 